\title{
NRAS NP_002515.1:p.G12S
}

National Cancer Institute

\section{Source}

National Cancer Institute. NRAS NP 002515.1:p.G12S. NCI Thesaurus. Code C155673.

A change in the amino acid residue at position 12 in the GT Pase NRas protein where glycine has been replaced by serine. 